\title{
Low-frequency Radio Absorption in Tycho's Supernova Remnant
}

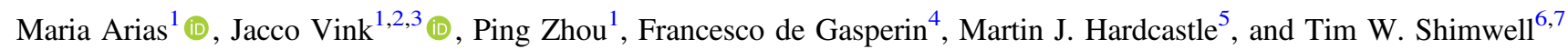 \\ ${ }^{1}$ Anton Pannekoek Institute for Astronomy, University of Amsterdam, Science Park 904,1098 XH Amsterdam, The Netherlands; M.AriasdeSaavedraBenitez@uva.nl \\ ${ }^{2}$ SRON, Netherlands Institute for Space Research, Sorbonnelaan 2, 3584 CA Utrecht, The Netherlands \\ ${ }^{3}$ GRAPPA, University of Amsterdam, Science Park 904, 1098 XH Amsterdam, The Netherlands \\ ${ }^{4}$ Hamburger Sternwarte, Universität Hamburg, Gojenbergsweg 112, D-21029, Hamburg, Germany \\ ${ }^{5}$ Centre for Astrophysics Research, School of Physics, Astronomy and Mathematics, University of Hertfordshire, College Lane, Hatfield AL10 9AB, UK \\ ${ }^{6}$ ASTRON, Netherlands Institute for Radio Astronomy, Oude Hoogeveensedijk 4, Dwingeloo, 7991 PD, The Netherlands \\ ${ }^{7}$ Leiden Observatory, Leiden University, P.O. Box 9513, 2300 RA Leiden, The Netherlands \\ Received 2019 August 29; revised 2019 October 17; accepted 2019 October 19; published 2019 December 2
}

\begin{abstract}
Tycho's supernova remnant (SNR) is the remnant of the SN Ia explosion SN1572. In this work we present new low-frequency radio observations with the LOw Frequency ARray (LOFAR) Low-band and High-band Antennae, centered at $58 \mathrm{MHz}$ and $143 \mathrm{MHz}$, and with an angular resolution of $41^{\prime \prime}$ and $6^{\prime \prime}$, respectively. We compare these maps to Very Large Array maps at $327 \mathrm{MHz}$ and $1420 \mathrm{MHz}$, and detect the effect of low-frequency absorption in some regions of the remnant due to the presence of free electrons along the line of sight. We investigate two origins for the low-frequency free-free absorption that we observe: external absorption from the foreground and internal absorption from Tycho's unshocked ejecta. The external absorption could be due to an ionized thin, diffuse cavity surrounding the SNR (although this cavity would need to be very thin to comply with the neutral fraction required to explain the remnant's optical lines), or it could be due to an over-ionized molecular shell in the vicinity of the remnant. We note that possible ionizing sources are the X-ray emission from Tycho, its cosmic rays, or radiation from Tycho's progenitor. For the internal absorption, we are limited by our understanding of the spectral behavior of the region at unabsorbed radio frequencies. However, the observations are suggestive of free-free absorption from unshocked ejecta inside Tycho's reverse shock.
\end{abstract}

Unified Astronomy Thesaurus concepts: Supernova remnants (1667); Interstellar plasma (851); Interstellar absorption (831); Molecular clouds (1072)

\section{Introduction}

Supernova remnants (SNRs) are the result of the interaction of a supernova explosion with its ambient medium. The X-ray and radio-bright shell characteristic of young SNRs is composed of a shocked ambient medium and stellar ejecta. Internal to the reverse shock there can be some stellar ejecta that have yet to encounter the reverse shock (McKee 1974). These ejecta were initially heated by the passage of the blast wave inside the star, but have since cooled due to adiabatic expansion. Because this material is internal to a shell bright in $\mathrm{X}$-rays and likely also in the UV, it can be photoionized. Several hundreds of years after the supernova event, the remnant still retains some imprint of the explosion; this is particularly the case for the unshocked ejecta.

SNRs have an effect on their surroundings, not only on the shocked ambient medium, but also on the still to-be-shocked neighborhood of the SNR. They are bright X-ray sources, as well as likely the sites of cosmic ray acceleration (Hillas 2005). Both the high-energy photons and the cosmic rays can deposit energy into the surroundings of the SNR, for instance, heating and ionizing nearby molecular clouds. Furthermore, during its lifetime and its pre-supernova (SN) stage, the progenitor star sculpts its ambient medium, for example, through stellar winds and ionizing radiation. The environment of the SNR is therefore a diagnostic of the star's pre-SN life, and of the SNR itself.

Tycho's SNR (SN 1572, G120.1+1.4, hereafter Tycho) is a young SNR, whose reverse shock might not have yet heated all of the stellar ejecta from the explosion. It is the result of a Type Ia event, as evidenced from the historical records of the light curve (Baade 1943), and from the optical spectrum as recovered from light echoes (Krause et al. 2008; Rest et al. 2008). From comparison of the X-ray spectra to hydrodynamical and spectral models, Badenes et al. (2006) concluded that the scenario that best fit the data is one in which $1.3 M_{\odot}$ of material were ejected at the time of the explosion into an ambient density of $\sim 0.6-3 \mathrm{~cm}^{-3}$. There is evidence that the density is higher in the northeast of the remnant, from $\mathrm{H} \alpha$ (Ghavamian et al. 2000), molecular gas (Lee et al. 2004; Zhou et al. 2016), and dust observations (Williams et al. 2013). The work of Woods et al. (2017) placed strict upper limits on the temperature and luminosity of Tycho's progenitor from the observed fraction of neutrals in the atomic gas, pointing to the merger of a double white dwarf binary as the most viable scenario for Tycho's SN explosion. On the other hand, the molecular shell found in Zhou et al. (2016) is more consistent with a single-degenerate scenario.

The remnant has been studied extensively, including at wavelengths that probe the unshocked ejecta. Lopez et al. (2015) observed it with NuStar, but they did not not detect any emission associated with the decay of radioactive ${ }^{44} \mathrm{Ti}$, point-like or extended. Gomez et al. (2012) observed it in the infrared with Herschel and Spitzer, and did not detect a cool dust component in the innermost region of unshocked ejecta, although they did not specifically look for line emission from photoionized, cold material. At low radio frequencies it has been observed with the Very Large Array (VLA) at $330 \mathrm{MHz}$ (Katz-Stone et al. 2000), and several times at $1.4 \mathrm{GHz}$ (Reynoso et al. 1997; Katz-Stone et al. 2000; Williams et al. 2016). It has also been observed at $660 \mathrm{MHz}$ with the Westerbork Synthesis Radio Telescope (WSRT; 


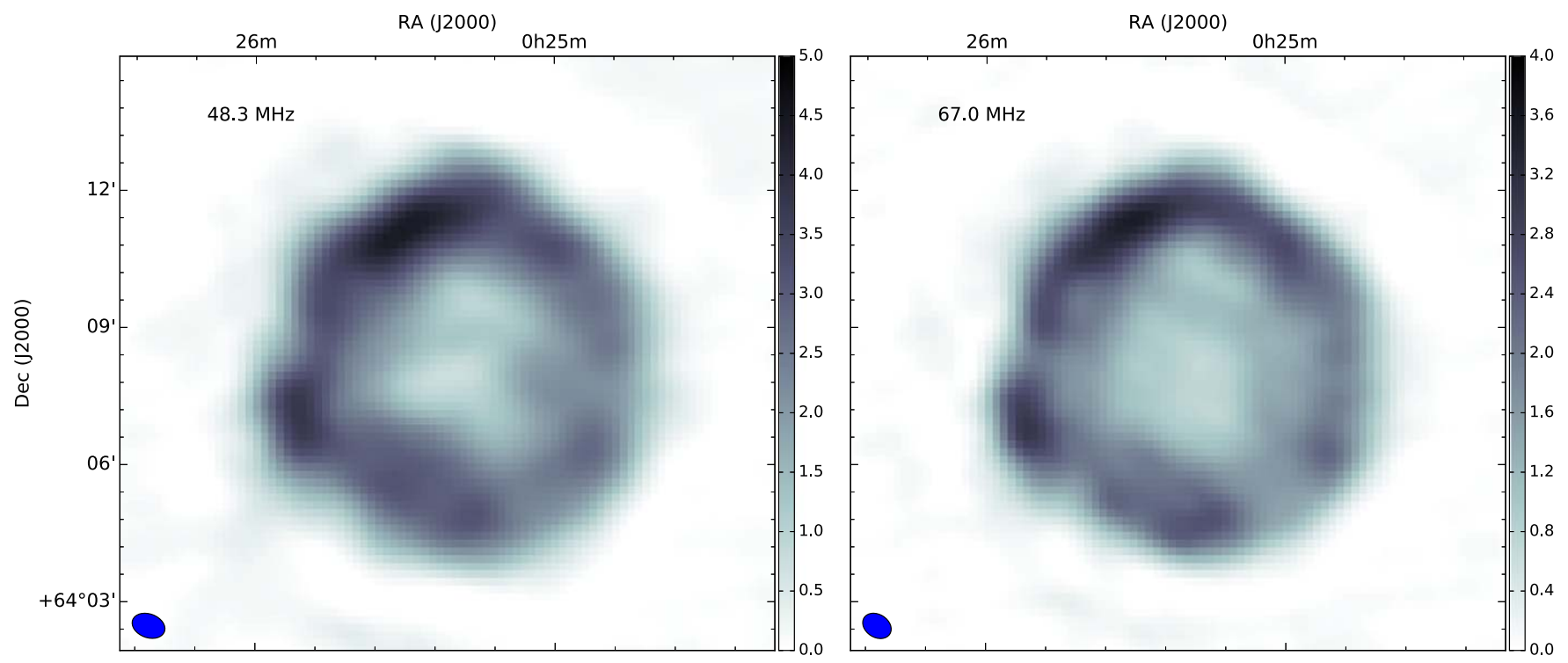

Figure 1. Tycho SNR as observed with the LOFAR LBA. The LBA bandwidth was split to make these two images, centered at $48.3 \mathrm{MHz}$ (left) and $67.0 \mathrm{MHz}$ (right), each $18 \mathrm{MHz}$ wide. The elliptical beam size is $41^{\prime \prime} \times 31^{\prime \prime}$, with position angle $56^{\circ}$, and the pixel size is $10^{\prime \prime}$ for both maps. The local rms noise is $0.03 \mathrm{Jy}^{-1} \mathrm{bm}^{-1}$ for the 67.0 MHz map and $0.08 \mathrm{Jy} \mathrm{bm}^{-1}$ for the $48.3 \mathrm{MHz}$ map. The flux density scale in both maps is in $\mathrm{Jy} \mathrm{bm}^{-1}$.

Duin \& Strom 1975), and, at lower resolution, at $408 \mathrm{MHz}$ as part of the Canadian Galactic Plane Survey (CGPS; Kothes et al. 2006).

In this paper we present new observations of Tycho with the LOw Frequency ARray (LOFAR; van Haarlem et al. 2013), both with the instrument's High-band Antenna (HBA; 120-168 $\mathrm{MHz}$ ) and the Low-band Antenna (LBA; 40-75 MHz). We compare these maps with higher frequency observations, and we detect localized free-free absorption from free electrons along the line of sight, from foreground material, and possibly also from material internal to the SNR reverse shock. We cannot use the measured absorption value to estimate how much mass there is in unshocked ejecta, although our results suggest that if unshocked material is present, it is in a combination of relatively highly ionized, cold, and significantly clumped states. The ionized ambient material could be either the diffuse cavity surrounding Tycho or its neighboring molecular clouds. Both scenarios have implications for the ionizing source.

\section{Observations and Data Reduction}

\subsection{Observations}

We observed Tycho's SNR with LOFAR under project LC10_011. The LBA observations were centered at R.A. = 00:25:21.5, decl. $=+64: 08: 26.9$, with a time on-source of 10 hr. The data were taken on 2018 May 18, in the LBA-Outer configuration, using 8 bit sampling, $1 \mathrm{~s}$ integration, and a frequency resolution of 64 channels per sub-band. The central frequency was $53.2 \mathrm{MHz}$, and the total bandwidth was 43.6 $\mathrm{MHz}$. A second beam was placed on calibrator 3C48 for the length of the observation.

For the HBA observations we made use of the possibility of co-observing with the LOFAR Two Metre Sky Survey (LoTSS; Shimwell et al. 2017). We identified the LoTSS pointing closest to Tycho, P007+64 (centered at R.A. = $00: 30: 40.8$, decl. $=+63: 36: 57.9$ ), and requested that it be observed during LOFAR cycle 10 as part of LC10_011. The observations were made with the standard LoTSS settings: $8 \mathrm{hr}$ on-source, $48 \mathrm{MHz}$ bandwidth, and an additional 10 minutes at the beginning and end of the observations to observe the calibrators (3C48 and 3C147, in this case).

\subsection{Low-band Antenna}

The LBA data were reduced with the LOFAR Lowfrequency Pipeline (de Gasperin et al. 2019). The pipeline calibrates the calibrator and transfers the solutions to the target, taking into account the main systematic effects in the LOFAR telescope, such as clock drift, polarization misalignment, ionospheric delay, Faraday rotation, ionospheric scintillation, beam shape, and bandpass.

Due to noise, we had to flag all the data at frequencies less than $40 \mathrm{MHz}$, as well as two LOFAR stations, CS013 and CS031. From the calibrator solutions we knew that there were very good ionospheric conditions during the observation, with almost no Faraday rotation (the calibrator was observed for the full duration of the observation, so we knew the ionosphere was good throughout). This allowed us to perform one round of self-calibration from our first image of the source, rather than from a sky model made at a different frequency.

The pipeline split the data into two frequency chunks, one centered at $48.3 \mathrm{MHz}$ and another centered at $67.0 \mathrm{MHz}$, which were imaged separately. We imaged the data with wsclean (Offringa et al. 2014), which allows for multi-scale, multi-frequency deconvolution with w-projections, and for applying the LOFAR beam. The visibilities were weighted with a Briggs parameter of zero (Briggs 1995). In order to filter out large-scale structure and in order to ensure common resolution among the maps, we used a $u-v$ range of 30-5000 $\lambda$. The two full-bandwidth LBA images centered at $48.3 \mathrm{MHz}$ and 67.0 MHz are shown in Figure 1.

In addition to the broadband maps, to search for spectral curvature, we made a series of narrow-band images, each $1.3 \mathrm{MHz}$ wide, centered at 40.1, 42.5, 44.8, 47.1, 49.5, 51.8, $54.2,56.5,58.9,61.2,63.5,65.8,66.9,68.1,70.5,72.8$, and 75.1 MHz. These maps were also made with a common $u-v$ range of $30-5000 \lambda$. 


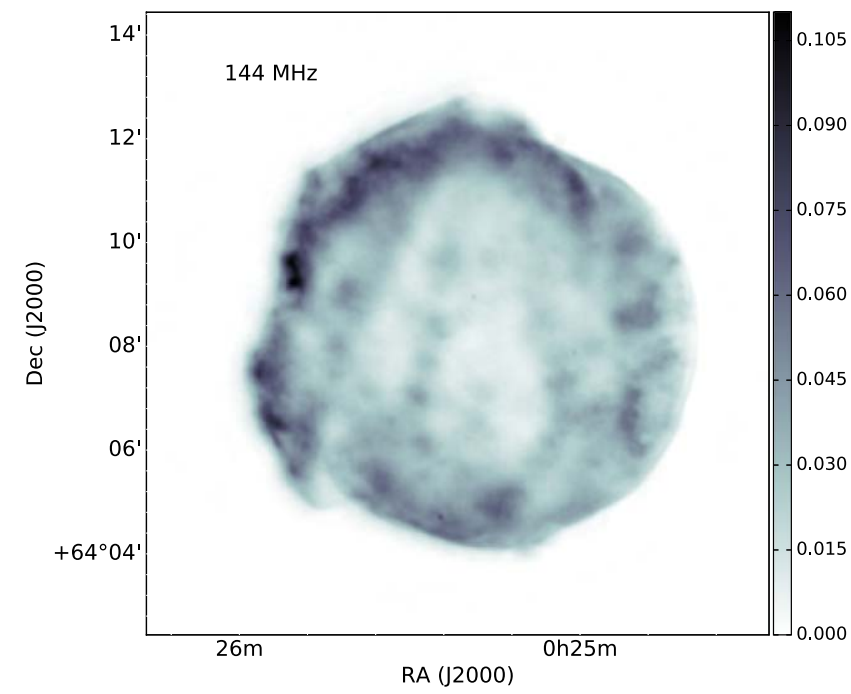

Figure 2. Tycho SNR as observed with the LOFAR HBA. The central frequency is $144 \mathrm{MHz}$, the bandwidth is $48 \mathrm{MHz}$, the beam size is $6^{\prime \prime}$, the pixel size is $1_{.}^{\prime \prime} 5$, and the local rms noise is $1 \mathrm{mJy} \mathrm{bm}^{-1}$. The flux density scale is in $\mathrm{Jy} \mathrm{bm}^{-1}$.

\subsection{High-band Antenna}

The HBA data were reduced in a direction-independent manner with the pre-facet calibration pipeline (van Weeren et al. 2016), which obtains diagonal solutions toward the calibrator and then performs clock-total electron content (TEC) separation, which distinguishes between clock offsets and drifts, and signal delays due to the electron column density in the ionosphere, and transfers the calibrator amplitudes and clock corrections to the data.

The calibrated data products were then imaged with the latest version of the ddf-pipeline ${ }^{8}$ (Shimwell et al. 2019; C. Tasse 2019 in preparation), which is the method used for reducing data from the LoTSS. The pipeline carries out several iterations of direction-dependent self-calibration, using DDFacet for imaging (Tasse et al. 2018) and KillMS for calibration (Tasse 2014a, 2014b; Smirnov \& Tasse 2015). The resulting HBA image is shown in Figure 2. The pipeline also produced three narrow-band images at 128, 144, and $160 \mathrm{MHz}$. The LOFAR HBA in-band spectral index is unreliable, but in order to use these narrow-band maps in our analysis we bootstrapped the maps to the expected flux densities of neighboring sources in the field, from the HBA broadband map.

\subsection{Archival Data}

We obtained the FITS files for the $327 \mathrm{MHz}$ VLA observation of Tycho carried out in 1991-1993 (Katz-Stone et al. 2000), as well as for the $1.4 \mathrm{GHz}$ VLA observation carried out in 2013-2015 (Williams et al. 2016). Katz-Stone et al. (2000) note that their map is sensitive to scales between $8^{\prime \prime}$ and $30^{\prime}$, which correspond to $114-25,800 \lambda \mathrm{s}$. The Williams et al. (2016) $L$-band map, combining the VLA A, B, C, and D configurations, is sensitive to scales between 1 !' 3 and $16^{\prime}$ $(212-15,800 \lambda s)$.

The integrated flux density of the $1382 \mathrm{MHz}$ map from Williams et al. (2016) is $41.7 \mathrm{Jy}$, and this is the value that we used for the analysis. However, if we directly measure the integrated flux density of the $327 \mathrm{MHz}$ image, it is $121.8 \mathrm{Jy}$.

\footnotetext{
Version 2.2, https://github.com/mhardcastle/ddf-pipeline/.
}

Table 1

Flux Densities of Tycho SNR

\begin{tabular}{lcccc}
\hline \hline $\begin{array}{l}\text { Freq } \\
(\mathrm{MHz})\end{array}$ & $\begin{array}{c}\text { Flux Density } \\
(\mathrm{Jy})\end{array}$ & $\begin{array}{c}\text { Error } \\
(\mathrm{Jy})\end{array}$ & Year & $\lambda$ Coverage \\
\hline 48.3 & 334 & 33 & 2018 & $30-5000 \lambda$ \\
67.0 & 275 & 27 & 2018 & $30-5000 \lambda$ \\
144.6 & 163 & 16 & 2018 & $50-50,000 \lambda$ \\
\hline 327 & 105.7 & 10.5 & 1995 & $114-25,800 \lambda$ \\
1382 & 41.7 & 4.2 & 2013 & $212-15,800 \lambda$ \\
\hline
\end{tabular}

Note. Observations at $327 \mathrm{MHz}$ and $1382 \mathrm{MHz}$ were taken with the VLA and are described by Katz-Stone et al. (2000) and Williams et al. (2016), respectively. See the discussion in Section 2.4 for $327 \mathrm{MHz}$ flux density.

This is $115 \%$ of the expected value for $S_{1 \mathrm{GHz}}=56 \mathrm{Jy}$ and $\alpha=0.58$ (Green 2017), and $117 \%$ for $S_{1 \mathrm{GHz}}=52.3 \mathrm{Jy}$ and $\alpha=0.63$, which are the best-fit values we find from a compilation of literature results (see the discussion in Section 3.1). We do not measure a level of background in the FITS image that accounts for this difference. Unfortunately, Katz-Stone et al. (2000) do not report the integrated flux density for their $327 \mathrm{MHz}$ observation.

Our analysis relies on the localized deviation from powerlaw behavior at low frequencies due to free-free absorption from ionized material along the line of sight (we discuss the method in detail in Section 3.2). The $327 \mathrm{MHz}$ and $1382 \mathrm{MHz}$ maps provide the fit with the information about the spectral behavior of the source when no absorption is present. If we take the flux density at $327 \mathrm{MHz}$ to be the $121.8 \mathrm{Jy}$ that we measure directly from the FITS file, we find it disproportionately affects the measured absorption, by setting an artificially high spectral index value for any given pixel, ${ }^{9}$ which then requires a much larger mass of absorbing material to account for the flux densities at LOFAR frequencies. For this reason, we normalized the flux density of the $327 \mathrm{MHz}$ map to $105.7 \mathrm{Jy}$, according to the best-fit power-law results for the compiled literature values as shown in Section 3.1.

When comparing interferometric maps, it is important to take into account the scales probed by the different instruments. When the emission is perfectly deconvolved, it is possible to compare higher resolution maps with lower resolution maps by simply smoothing them to a common resolution. However, the short baseline $u-v$ coverage matters if interferometers do not probe the same scales, especially for Galactic observations, for which the sources might be embedded in large-scale diffuse emission.

We summarize the $u-v$ scales probed by the maps used in our analysis in Table 1. Our LOFAR maps are sensitive to large angular scales, which might result in additional large-scale continuum emission that is resolved out by the VLA maps. This would result in a spectral index steepening. We note this issue as a possible source of error.

\section{Results \\ 3.1. Total Flux Density}

We report the total flux density of Tycho as seen with the LOFAR telescope LBA and HBA in Table 1. We also include

\footnotetext{
9 The $121.8 \mathrm{Jy}$ and $41.7 \mathrm{Jy}$ values at $327 \mathrm{MHz}$ and $1382 \mathrm{MHz}$ correspond to a spectral index of $\alpha_{327 / 1382}=0.74$, much higher than the overall spectral index of the source.
} 


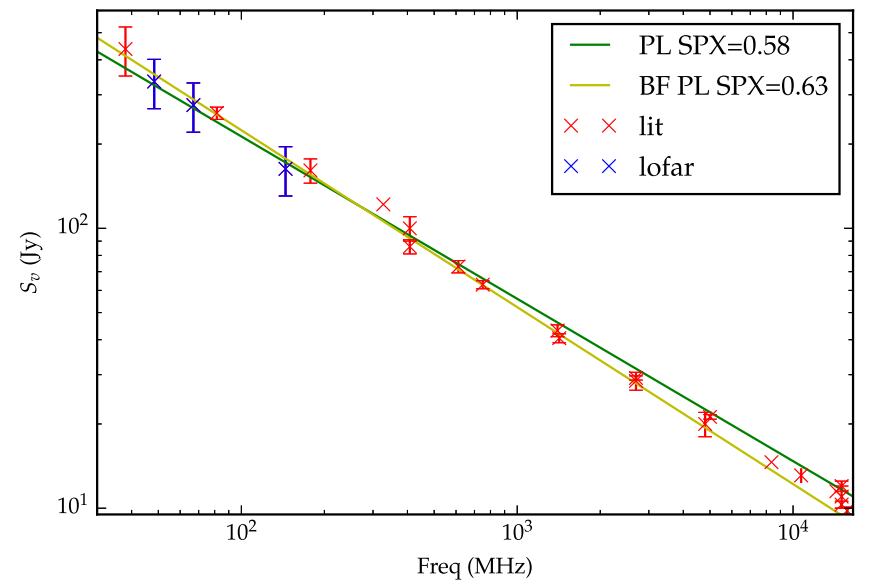

Figure 3. Radio spectrum of Tycho, including measurements from this work (in blue). The green line corresponds to the power-law spectral index (PL SPX) of 0.58 reported in Green (2017), and the yellow line is the best-fit (BF) powerlaw spectral index from these data points. The literature (lit) values in red are taken from: Klein et al. (1979), Green et al. (1975), Hurley-Walker et al. (2009), Katz-Stone et al. (2000), Kothes et al. (2006), Planck Collaboration et al. (2016), Gao et al. (2011), Langston et al. (2000), Williams et al. (1966), Scott \& Shakeshaft (1971), Artyukh et al. (1969), Bennett (1963), Fanti et al. (1974), Conway et al. (1965), Kellermann et al. (1969), and Horton et al. (1969).

the values from the $327 \mathrm{MHz}$ and $1382 \mathrm{MHz}$ VLA observations (Katz-Stone et al. 2000; Williams et al. 2016) which we relied on for the analysis.

We compiled a series of radio flux densities in the literature, and plotted the LOFAR values alongside them (Figure 3). Fitting a function of the form $S_{\nu}=S_{1 \mathrm{GHz}}\left(\frac{\nu}{1 \mathrm{GHz}}\right)^{-\alpha}$ gives a best fit of $S_{1 \mathrm{GHz}}=52.3 \pm 2.0 \mathrm{Jy}$ and $\alpha=0.63 \pm 0.02$, whereas the value listed in the Green SNRs catalog is $S_{1 \mathrm{GHz}}=56 \mathrm{Jy}$ and $\alpha=0.58$ (Green 2017).

The systematic calibration errors in the LOFAR flux scale are of the order of $10 \%$, which dominates the uncertainties, rather than the noise. For this reason we take $10 \%$ errors when we report the integrated flux densities of Tycho in the broadband images in Table 1 and in Figure 3. However, the $10 \%$ errors are on the total flux scale rather than the disagreement between in-band measurements. They are therefore an overestimate for the purposes of our analysis (our fits result in residuals that are much smaller than the error bars). The fact that we do not know the statistical errors of the flux densities presents an issue for the analysis.

In order to solve this problem, we artificially shrank the error bars of the LOFAR images (see Figure 4) until the reduced $\chi^{2}$ of the best-fit power law for these points was 1 . This provides us with a more meaningful estimate of the errors in our pixelby-pixel analysis.

The flux densities of the LBA narrow-band maps are plotted in Figure 4. If we only consider the LOFAR LBA and HBA results, we measure a steeper spectral index than when we take into account measurements at higher frequencies $(\alpha=0.67$ instead of $\alpha=0.58$ or $\alpha=0.63$ ). The best-fit value of $\alpha$ for the LOFAR points $(\alpha=0.63)$ results in a $\Delta \chi^{2}=23.7$ improvement over the fixed $\alpha=0.58$ scenario, for one additional degree of freedom.

\subsection{Model Parameters: External Absorption}

A synchrotron source with spectrum $S_{\nu} \propto \nu^{-\alpha}$ that is subject to free-free absorption from cold, ionized, interstellar medium

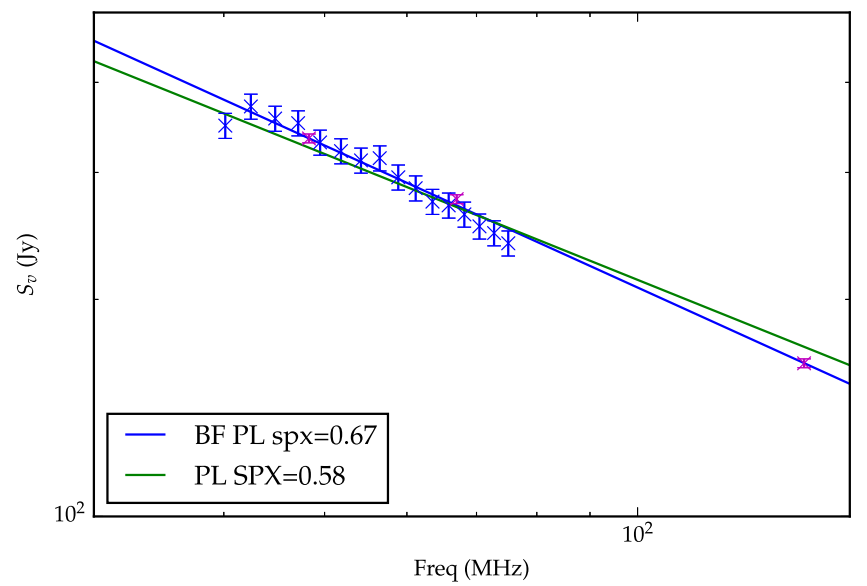

Figure 4. Radio spectrum of Tycho at LOFAR frequencies. The magenta points correspond to the full bandwidth maps and the blue points correspond to the narrow band maps. The green line corresponds to the power-law spectral index (PL SPX) of 0.58 reported in Green (2017), and the blue line is the bestfit $(\mathrm{BF})$ power-law spectral index from the LOFAR data points. The errors bars have been normalized so the reduced $\chi^{2}$ of the best-fit power law (in blue) is equal to 1 , but we note that the uncertainties in the LOFAR in-band have not been systematically analyzed and can be unreliable. Our measurements agree with earlier reports that the radio spectrum of Tycho steepens at low radio frequencies.

(ISM) material along the line of sight results in the following radio spectrum:

$$
S_{\nu}=S_{0}\left(\frac{\nu}{\nu_{0}}\right)^{-\alpha} e^{-\tau_{\nu, \mathrm{ISM}}}
$$

where (Rybicki \& Lightman 1979)

$$
\tau_{\nu}=3.014 \times 10^{4} Z\left(\frac{T}{\mathrm{~K}}\right)^{-3 / 2}\left(\frac{\nu}{\mathrm{MHz}}\right)^{-2}\left(\frac{E M}{\mathrm{pc} \mathrm{cm}^{-6}}\right) g_{\mathrm{ff}},
$$

$Z e$ is the charge of the free-free absorbing ions, $T$ is the temperature of the plasma, $E M \equiv \int_{0}^{s} n_{\mathrm{e}}^{2} d s^{\prime}$ is the emission measure, $n_{\mathrm{e}}$ is the number density of electrons, and $g_{\mathrm{ff}}$ is a Gaunt factor, given by

$$
g_{\mathrm{ff}}=\left\{\begin{array}{c}
\ln \left[49.55 Z^{-1}\left(\frac{\nu}{\mathrm{MHz}}\right)^{-1}\right]+1.5 \ln \frac{T}{\mathrm{~K}} \\
1 \quad \text { for } \frac{\nu}{\mathrm{MHz}} \gg\left(\frac{T}{\mathrm{~K}}\right)^{3 / 2} .
\end{array}\right.
$$

We convolved all the images to a resolution of $41^{\prime \prime}$, and performed a pixel-by-pixel fit (with a pixel size of $10^{\prime \prime}$ ) to Equation (1). The results are plotted in Figure 5. For each pixel, we fitted for an amplitude $S_{0}$, the spectral index $\alpha$, and the optical depth for the ISM material at $40 \mathrm{MHz} \tau_{40, \text { ISM. As }}$ errors, we plot the diagonal term of the covariance matrix corresponding to each parameter.

We also show the fit results for three integrated regions that show external absorption (see Figure 5, right panel): the region toward the northeast, the absorbed region in the center, and the whole rim of the SNR. These regions are labeled in Figure 6, and their spectral energy distribution (SED) along with the best-fit results are shown. The parameters $\alpha$ and $\tau_{40 \text {,ext }}$ are correlated (see contour plots in Figure 7), but for two of the three regions we require absorption at the $3 \sigma$ level or higher. 

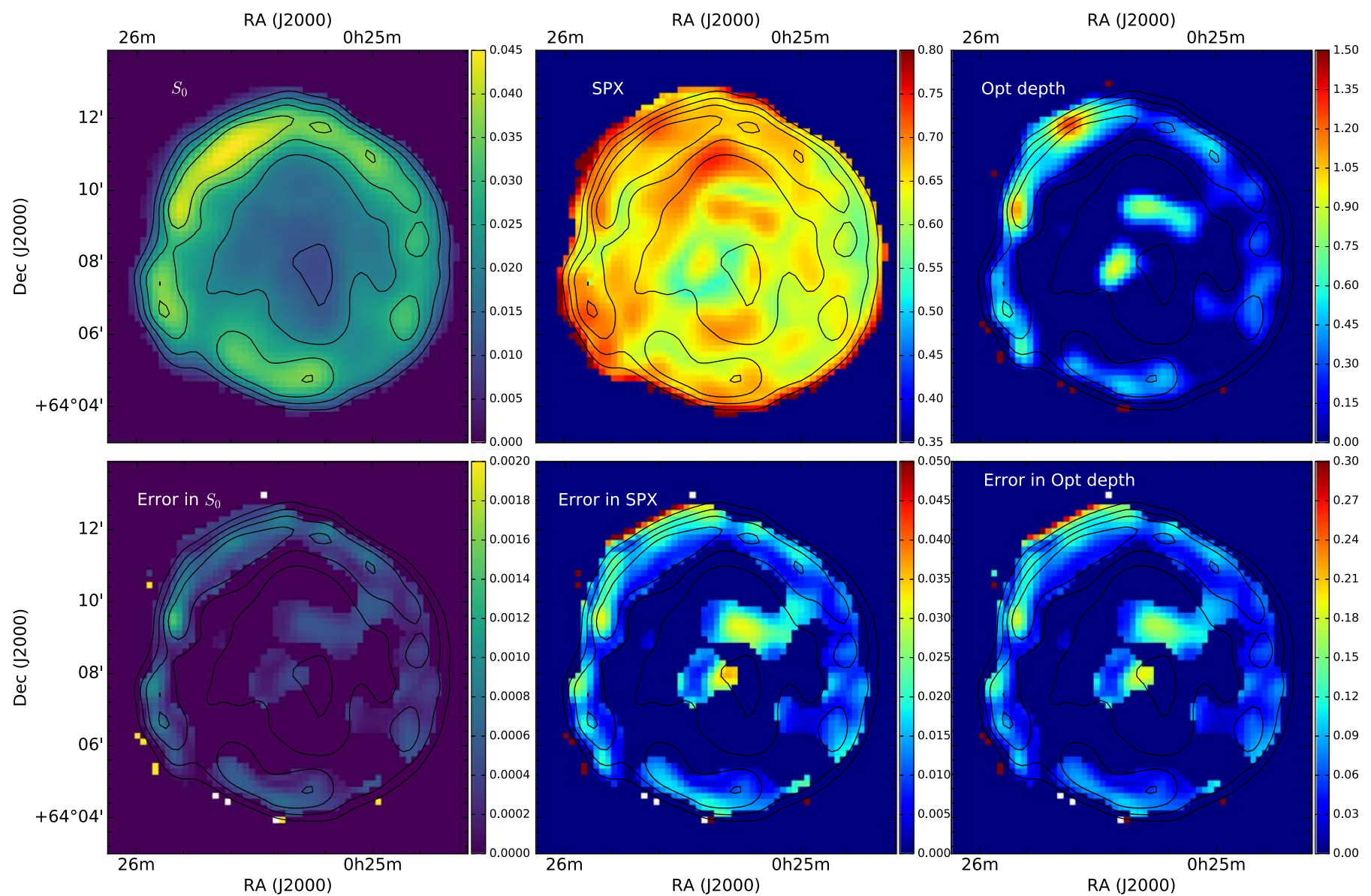

Figure 5. Results of fitting Equation (1) to the maps. For each pixel we fitted for amplitude $S_{0}$, the spectral index $\alpha$, and the optical depth for the ISM material at $40 \mathrm{MHz} \tau_{40, \mathrm{ISM}}$. The units of the $S_{0}$ map on the left are $\mathrm{Jy} \mathrm{bm}^{-1}$. The errors are the diagonal term of the covariance matrix corresponding to each parameter.

\subsection{Model Parameters: Internal Absorption}

A synchrotron source that is subject to internal free-free absorption from its cold, ionized, unshocked ejecta will have a dimming factor that goes as $\left(f+(1-f) e^{-\tau_{\nu}}\right)$, where $f$ is the fraction of the synchrotron emission that is produced by the front side of the shell and, therefore, cannot be absorbed by its internal material. This factor multiplies Equation (1) resulting in the following radio spectrum:

$$
S_{\nu}=S_{0}\left(\frac{\nu}{\nu_{0}}\right)^{-\alpha}\left(f+(1-f) e^{\left.-\tau_{\nu, \mathrm{int}}\right)} e^{-\tau_{\nu, \mathrm{ISM}}}\right.
$$

Internal free-free absorption can only occur in the region inside the projected reverse shock, since there cannot be unshocked absorbing material outside the reverse shock.

Warren et al. (2005) found the reverse shock in Tycho's SNR to have a radius of $183^{\prime \prime}$ and center R.A. $=0: 25: 19.40$, decl. $=$ $+64: 08: 13.98$, from a principal component analysis of the X-ray data. We measured the flux density for each image for the region internal to the reverse shock, with the aim to look for internal absorption. We do not find any external absorption in the region internal to the reverse shock, save for two clumps in the center of the SNR (Figure 5), and so, to simplify our fit, we removed the area of absorption in the center (the blue region in Figure 6) from our area of internal absorption (the yellow region in Figure 6), and just fitted for an amplitude, the parameter $f$, and an internal optical depth,

$$
S_{\nu}=S_{0}\left(\frac{\nu}{\nu_{0}}\right)^{-\alpha}\left(f+(1-f) e^{\left.-\tau_{\nu, \text { int }}\right)}\right.
$$

As described in Section 3.1, we rescaled the error bars in such a way that the reduced $\chi^{2}$ of the best-fit power law $\left(S_{\nu}=S_{0}\left(\frac{\nu}{\nu_{0}}\right)^{-\alpha}\right.$; with no absorbing component) was 1 . The best-fit power law for this region corresponds to $\alpha=0.63$. From here, we compared how including an internal absorbing component improved the fit.

Setting $f=0.5$ (that is, fixing the synchrotron emission such that half comes from the back and half comes from the front of the shell) gives a best fit of $\alpha=0.63, \tau_{40, \text { int }}=3 \times 10^{-8}$. This means that the best-fit value for internal absorption with $f=0.5$ corresponds to no internal absorption. Setting $f=0.5$, for $\tau_{40, \text { int }}=0.11$ we obtained a $\Delta \chi^{2}=4$ (with respect to the best-fit result). We take this to be the $2 \sigma$ upper limit estimate on the internal optical depth, and so in the internal emission measure $E M_{\text {int }}$ (for $T=100, Z=3$ ).

Alternatively, if we fit a region that shows internal absorption with a power law, the spectral index flattens due to the presence of absorption. Katz-Stone et al. (2000) found $\alpha=0.71$, rather than $\alpha=0.63$ for this region, from a $330 \mathrm{MHz}$ to $1.4 \mathrm{GHz}$ spectral index study. In fact, the higher frequency data points, where no absorption is present, should be the ones that determine the spectral index. At low frequencies the original spectral index should be recovered, 


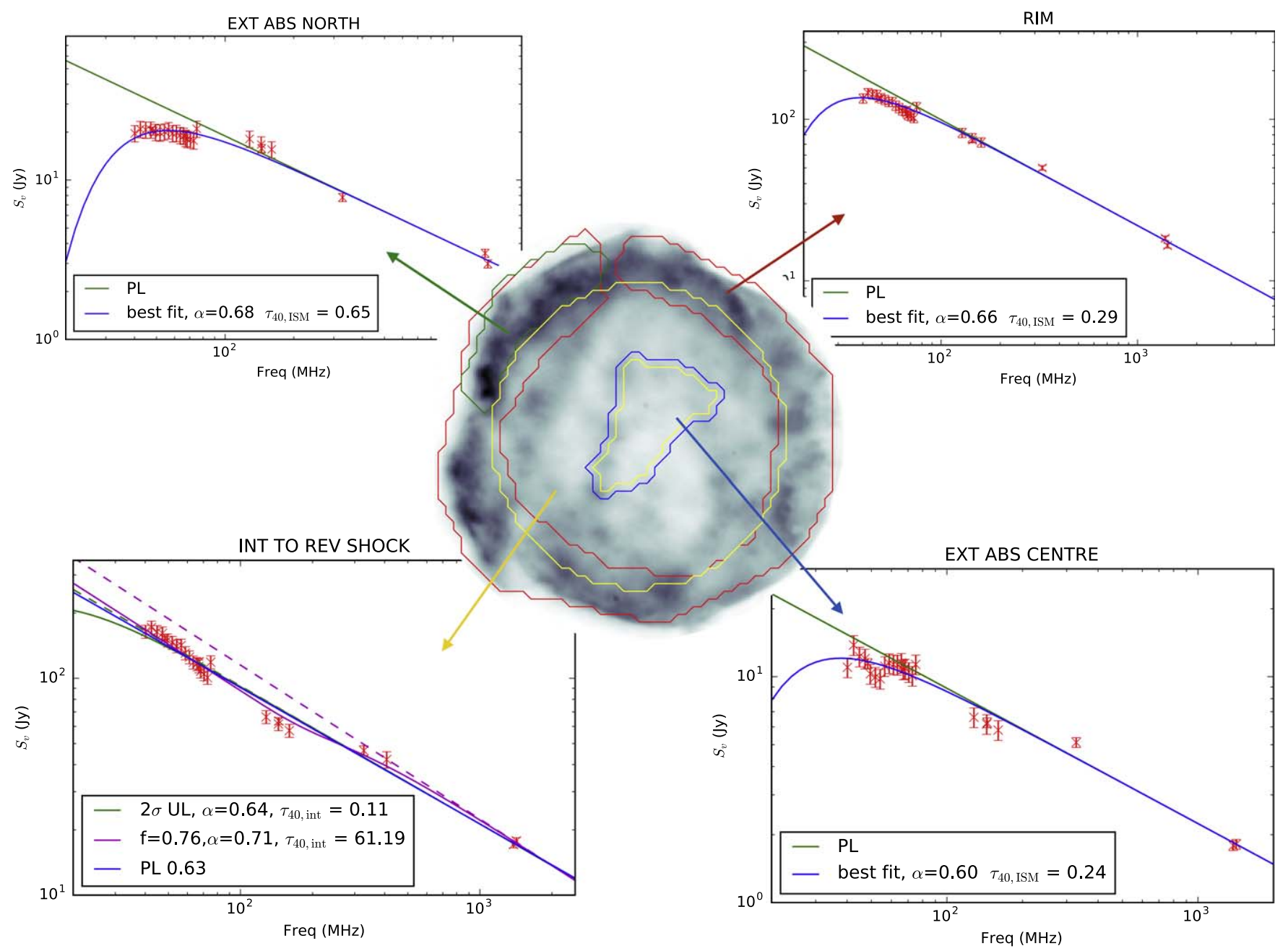

Figure 6. HBA map with overlaid regions of analysis. The values of $f, \tau_{40}$, and $\alpha$ are unitless. For all regions, the errors were rescaled in such a way that the best-fit power law has a reduced $\chi^{2}$ of 1 . The top plots and the bottom right plot (corresponding to the green, red, and blue regions as overlaid on Tycho) are fitted including external absorption (in blue, the best-fit unabsorbed power law is in green), and in all cases including the absorption term improves the fit: with a $\Delta \chi^{2}=16$ for "EXT ABS NORTH", a $\Delta \chi^{2}=4$ for "EXT ABS CENTRE", and a $\Delta \chi^{2}=10.5$ for "RIM" (in all cases, for an additional degree of freedom). The bottom left plot corresponds to the region of possible internal absorption. The mask of the reverse shock radius is plotted in yellow over the map of Tycho. In the legends, "UL" stands for upper limit and "PL" stands for power law.

but with the amplitude dimmed by a factor of $f$. Hence, we fixed the spectral index to $\alpha=0.71$ and fitted for the remaining parameters. This results in a very high value of the optical depth, $\tau_{40 \text {,int }}=61$.

The results of our fits (power law, internal absorption, $2 \sigma$ upper limit in internal absorption, and $\alpha$ fixed to the value given by Katz-Stone et al. 2000) are tabulated in Table 2. We also plotted the results for the power-law fit (in blue), the upper limit to the $E M$ (for $T=100, Z=3$; in green), and the fixed $\alpha$ (in magenta; dashed lines indicate the unabsorbed flux density) in Figure 6, bottom left corner. Here we show the rescaled errors rather than the original error bars.

From Table 2, fixing $\alpha=0.71$ and adding an absorbing component does seem to significantly improve the fit (the fact that the reduced $\chi^{2}$ is equal to 0.4 would normally suggest overfitting, but in this case the reduced $\chi^{2}$ of the power-law fit was artificially set to 1$)$. The required emission measure is unphysical (see the discussion in Section 4.4), but it is very sensitive to the choice of $\alpha$ and $f$. We cannot confidently claim a detection of unshocked ejecta in Tycho's SNR because of our limited knowledge of the errors in the flux densities, and because of the degeneracy of the parameters, but our data are suggestive that there is indeed some unshocked material inside Tycho's reverse shock. ${ }^{10}$

In order to better estimate the $E M$ due to internal absorption we need more high-frequency data points in the few gigahertz range that can unambiguously determine the unabsorbed flux density and spectral index for this region. Additional observations in the few hundred megahertz range would help better model the curvature due to the free-free absorption, and, if it were ever possible, observations at even lower frequencies would further discriminate between the different models. In this work we are relying on only the points at $327 \mathrm{MHz}$ and $1382 \mathrm{MHz}$ for information about the unabsorbed flux density and spectrum, and the $327 \mathrm{MHz}$ map was rescaled (see the discussion in Section 2.4). Moreover, the behavior of the LOFAR in-band seems to be pushing the data point in a steeper

\footnotetext{
${ }^{10}$ In the conference Supernova Remnants: An Odyssey in Space after Stellar Death II (Chania, Greece, 2019 June) we presented preliminary results of a very high EM detection from Tycho's unshocked ejecta (http://snr2019.astro. noa.gr/wp-content/uploads/2019/08/D3-0940-Arias.pdf). This was due to us not noticing at first that the $330 \mathrm{MHz}$ map had a very high flux density value, which steepened the best-fit spectral index, and thus the required amount of absorbing material.
} 

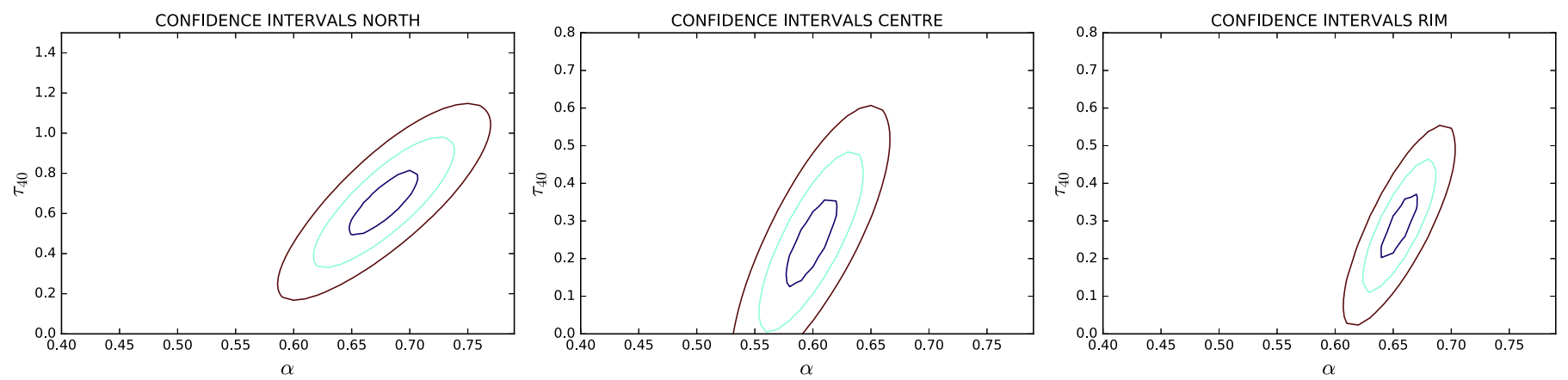

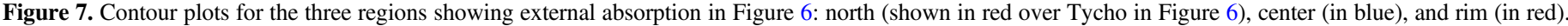

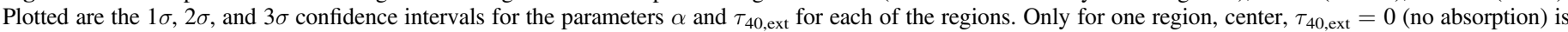

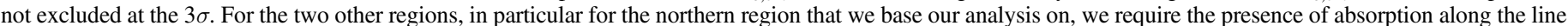
of sight at the $3 \sigma$ level.

Table 2

Fits to the Region Internal to the Reverse Shock

\begin{tabular}{lccccc}
\hline \hline Fit & $\alpha$ & $f$ & $\tau_{40, \text { int }}$ & red $\chi^{2}$ & $\Delta \chi^{2}$ \\
\hline PL & 0.63 & $\ldots$ & $\ldots$ & 1.0 & $\ldots$ \\
Best-fit int abs & 0.63 & $0.5^{*}$ & $3 \times 10^{-8}$ & 1.1 & 0 \\
UL in int abs & 0.64 & $0.5^{*}$ & 0.11 & 1.3 & 4 \\
Fixed $\alpha$ & $0.71^{*}$ & 0.76 & 61.1 & 0.4 & 16 \\
\hline
\end{tabular}

Note. The best-fit emission measure $E M$ assumes $T=100 \mathrm{~K}$ and $Z=3$. Parameterized, it corresponds to $E M=E M_{\text {table }} \mathrm{pc} \mathrm{cm} \mathrm{cm}^{-6}\left(\frac{g_{\mathrm{ff}}(T=100, Z=3)}{g_{\mathrm{ff}}(T / 100 \mathrm{~K}, Z / 3)}\right) \times$ $\left(\frac{Z}{3}\right)\left(\frac{T}{100 \mathrm{~K}}\right)^{-3 / 2}$. The reduced $\chi^{2}$ to the power-law fit is 1 by definition. Values indicated with ${ }^{*}$ are fixed, not fitted for. The $\Delta \chi^{2}$ for the Fixed $\alpha$ model is with respect to the power-law model "PL," corresponding to two additional degrees of freedom. The upper limit "UL" was derived as discussed in the text.

spectral index direction. For this reason, observations that increase the leverage arm in frequency would allow us to better constrain the amount of $E M$ due to unshocked material.

Having said that, the integrated flux densities as measured by LOFAR are in line with what we expect from the literature. There are some regions where the maps can have artifacts, but the flux densities that we are considering in this section are taken from the yellow region in Figure 6, which is much larger than the resolution of any given map. Moreover, the LBA and the HBA data both show the effect of absorption, even though the two LOFAR antennas are effectively different instruments, and the data were reduced with two independent pipelines.

\section{Discussion}

\subsection{Spectral Index}

Katz-Stone et al. (2000) carried out a study of Tycho's spectral index at low radio frequencies $(330 \mathrm{MHz}$ and $1.5 \mathrm{GHz})$, and found that Tycho has localized spectral variations with regions as flat as $\alpha=0.44$ and as steep as $\alpha=0.72$. Our best-fit spectral index map (middle panel in Figure 5) shows values within this range, and, in a few cases, slightly higher values, $\alpha \lesssim 0.8$.

Duin \& Strom (1975) reported a significant steepening of the spectrum near the center of the SNR and suggested that particles near the boundary might be accelerated with a flatter spectrum, but Klein et al. (1979) did not find steepening in their observations at $10 \mathrm{GHz}$. We do not find a steepening coincident with the center of the remnant, but rather we find the spectrum of the western and north-western region of the remnant to be steeper than the rest.

The question of whether Tycho has a curved spectrum has been discussed in the literature. Roger et al. (1973) modeled Tycho's integrated radio spectrum with two power-law components (which results in a locally concave spectrum), Reynolds \& Ellison (1992) modeled it with a non-linear shock model of first-order Fermi acceleration and found agreement with a concave-up synchrotron spectrum, whereas Vinyaikin et al. (1987) found that a single power law can describe the radio spectrum at these frequencies. As we discussed in Section 3.1, the LOFAR data points do show a steeper spectral behavior than expected, although the in-band response of the LOFAR LBA has not been systematically analyzed, and is not yet reliable.

\subsection{External Absorption}

In order to convert the value of optical depth in Figure 5 into a quantity that allows us to derive physical properties of the gas we use Equation (2), from which we obtain an emission measure value, $E M_{\text {ISM. }}$. The emission measure depends on the temperature and ionization state of the plasma. The ISM has a wide range of temperatures, from $\sim 10 \mathrm{~K}$ in molecular clouds to $\sim 10,000 \mathrm{~K}$ in the warm ionized medium (Draine 2011). We therefore provide three emission measure maps in Figure 8, assuming $T=10 \mathrm{~K}, T=100 \mathrm{~K}$, and $T=10,000 \mathrm{~K}$, to aid our discussion in the current section. Since the ISM is primarily composed of hydrogen, for all three maps we assume $Z=1$.

The region to the northeast with the high emission measure value (the region in green in Figure 6) seems to match the position of a molecular cloud found in Lee et al. (2004) and Zhou et al. (2016), seen most clearly in Figure 1 of the latter paper at velocities between -62 and $-66 \mathrm{~km} \mathrm{~s}^{-1}$. At these velocities there are also multiple structures that coincide in position with the rim of the source, which our fit also identifies as having free-free absorption. The region in the northeast of the remnant where we find the highest values of the $E M_{\text {ISM }}$ also coincides with the region of high $\mathrm{HI}$ absorption seen in Reynoso et al. (1999). The region in the center of Tycho has some morphological coincidence with the molecular structure seen at $-56 \mathrm{~km} \mathrm{~s}^{-1}$ in Zhou et al. (2016), although the similarity is not striking, and there does not seem to be any associated neutral hydrogen structure. Our method traces ionized material, which one does not expect in molecular clouds but could be present at their outer boundary, so it is not 

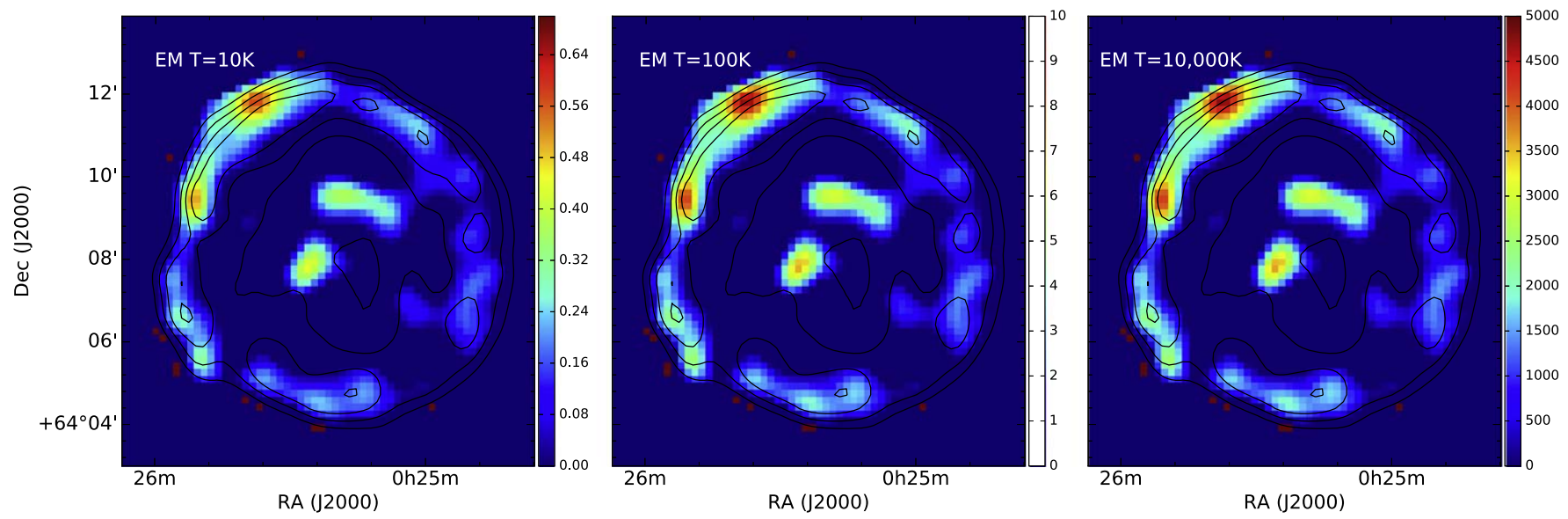

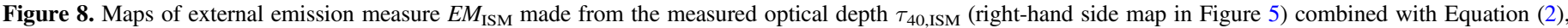

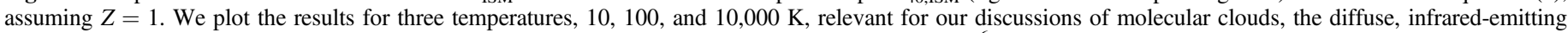
medium around Tycho, and the ISM warm ionized gas, respectively. The units of $E M_{\mathrm{ISM}} \operatorname{are~} \mathrm{pc} \mathrm{cm}^{-6}$.

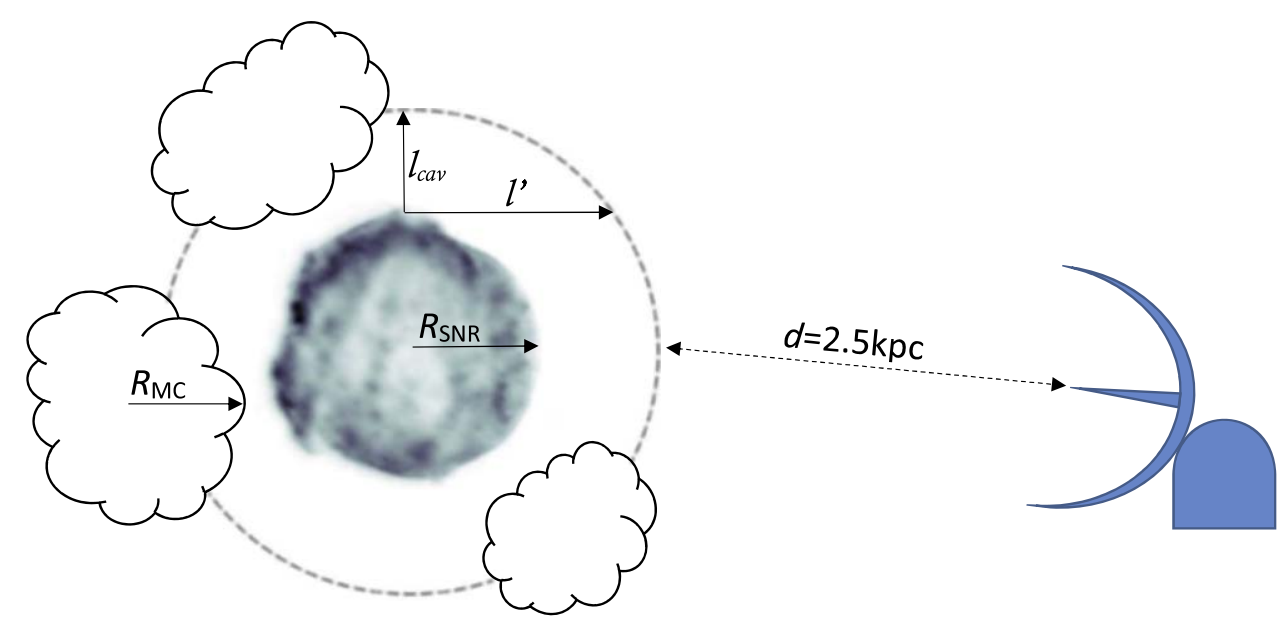

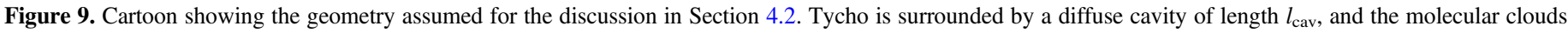
are in a ring-like shape around it.

necessary that our measured $E M_{\mathrm{ISM}}$ matches the structure of molecular/neutral material in detail.

The scale and distance of the ionized features are not straightforward from these observations. Tycho is the background synchrotron source, so the ionized material must be in front of it, but in principle it could be local to Tycho, unrelated ISM, or a combination of the two (although it would be a big coincidence if one of the two did not have a dominant effect).

We know from Zhou et al. (2016) that Tycho is likely inside an expanding wind bubble that is sweeping up molecular material. We depict the structure we assume for our analysis in a cartoon in Figure 9. The remnant is surrounded by, but its shock is still not interacting with, molecular clouds. This means that there is a cavity of thickness $l$ (and radius $R_{\mathrm{SNR}}+l$ ) of lowdensity material $\left(\rho=0.1-0.2 \mathrm{~cm}^{-3}\right.$; Williams et al. 2013), surrounded by dense molecular material with an average density of $10^{2}-10^{3} \mathrm{~cm}^{-3}$ (Zhou et al. 2016).

We will consider three possibilities: (1) that the ionized material we see in Figure 5, right-hand side, is due to ionized material along the line of sight, unrelated to Tycho; (2) that it is the low-density cavity material that is ionized; and (3) that the molecular clouds are responsible for the free-free absorption. In Section 4.3 we briefly mention possible ionizing sources.
1. Ionized ISM. Hwang et al. (2002) tabulated the $N_{\mathrm{H}}$ as measured from Chandra data, toward Tycho, and found values ranging from $N_{\mathrm{H}}=(5.3-7.5) \times 10^{21} \mathrm{~cm}^{-2}$, depending on the model employed.

For the region in green in Figure 6 the optical depth at $40 \mathrm{MHz}$ is $\tau_{40, \text { ISM }}=0.65$, which corresponds to an emission measure of $E M=0.30 \mathrm{pc} \mathrm{cm}^{-6}$ for $T=10 \mathrm{~K}$ and $E M=2469 \mathrm{pc} \mathrm{cm}^{-6}$ for $T=10,000 \mathrm{~K}$. Since $E M=n_{\mathrm{e}}^{2} l$, $N_{\mathrm{H}}=\mathrm{n}_{\mathrm{H}} l$, and $n_{\mathrm{e}}=\chi_{\mathrm{e}} \mathrm{n}_{\mathrm{H}}$ (where $\chi_{\mathrm{e}}$ is the ionization fraction, $0 \leqslant \chi_{\mathrm{e}} \leqslant 1$ ), then, using $l=d$, the distance to Tycho, we find that the required ionization fraction of the intervening ISM is $\chi_{\mathrm{e}}=\frac{\sqrt{E M l}}{N_{\mathrm{H}}} \sim 0.015 \sqrt{\frac{l}{2.5 \mathrm{kpc}}}$ for $T=10 \mathrm{~K}$, or alternatively, $\chi_{\mathrm{e}} \sim 1.35 \sqrt{\frac{l}{2.5 \mathrm{kpc}}}$ for $T=10,000 \mathrm{~K}$. The $10,000 \mathrm{~K}$ assumption for the diffuse ISM gas is more reasonable than the $10 \mathrm{~K}$ (Draine 2011), although, of course, this gas does not extend evenly along the line of sight to Tycho, but is likely in a patchy distribution (which would lower $\chi_{\mathrm{e}}$ to a more reasonable value). We do not know the relative depths of these warm ionized gas along the line of sight to Tycho, so unfortunately we cannot constrain a $\chi_{\mathrm{e}}$ for the case of this ISM.

Another point to note is that $\tau_{40, \text { ISM }}=0.65$ corresponds to an optical depth of $\tau_{30.9}=1.2$ at $30.9 \mathrm{MHz}$, although this is 
for a very small area $\left(3.6 \operatorname{arcmin}^{2}\right)$. Kassim (1989) studied optical depths toward 15 Galactic SNRs, and found only one source with $\tau_{30.9}>1$. The integrated radio spectrum of Tycho (Figure 3) shows no indication of free-free absorption from the ISM kicking in at frequencies lower than $100 \mathrm{MHz}$. There is a slight drop visible in the spectrum from LOFAR narrow-band maps (Figure 4), although this relies only on the data point at $40 \mathrm{MHz}$. For the integrated spectrum of Tycho's SNR we measure a best fit of $\tau_{30.9}=0.1$, well on the low side of the values measured by Kassim (1989).

The relatively high value of the optical depth in the region in green in Figure 6 and its small area suggest that this is a small clump of ionized material. We cannot know if the clump is relatively close to the source or somewhere along the line of sight.

Finally, the low-frequency absorption is only seen in a ringlike structure in the rim of the SNR and in two clumpy regions in the SNR center. In the remaining regions in the interior we do not find any detectable absorption. It is unlikely, though, that the foreground ISM gas has the shape we see over Tycho, with a clear ring and a mostly empty interior. The regular morphology seen in the maps in Figure 8 does not favor the ionized ISM scenario as the dominant source of absorption.

2. Ionized diffuse cavity surrounding Tycho. Consider that it is the cavity surrounding Tycho that is responsible for the ionization we see at LOFAR frequencies.

The size of the ionized cavity may influence the distributions of the foreground absorption. As shown in Figure 9, the depth of the ionized materials $l^{\prime}$ is as a function of the projection radius $r$ ( $r=0$ at the SNR center, $r=R$ at the SNR boundary), the radius of the SNR $R$ and the thickness of the cavity $l$ $\left(l^{\prime}=\sqrt{l^{2}+2 l R}\right)$, resulting in

$$
\begin{gathered}
l^{\prime}(R) \approx \begin{cases}\sqrt{2 R l}, & \text { if } l \ll R \\
l, & \text { if } l \gg R\end{cases} \\
l^{\prime}(0)=l .
\end{gathered}
$$

If the cavity size is much larger than the SNR radius, we would see a uniform ionization distribution as $l^{\prime}(r)=l$. The ring-like ionization distribution suggests that the cavity is small and might be close to the SNR radius.

Williams et al. (2013) found that the ISM density around Tycho is only $n_{\mathrm{H}}=0.1-0.2 \mathrm{~cm}^{-3}$, and that there is dust with a temperature of $T=100 \mathrm{~K}$.

The optical depth value we report for the rim of Tycho (the region in red in Figure 6), $\tau_{40, \text { ISM }}=0.29$, assuming $Z=1$ and $T=100 \mathrm{~K}$, corresponds to an emission measure of $E M=2.1$ pc $\mathrm{cm}^{-6}=n_{\mathrm{e}}^{2} 1_{\text {cav }}$, where $l_{\text {cav }}$ is the size of the cavity. This implies $n_{\mathrm{e}}=1.5 \sqrt{\frac{l_{\mathrm{cav}}}{1 \mathrm{pc}}} \mathrm{cm}^{-3}$. Recall that $n_{\mathrm{e}}=\chi_{\mathrm{e}} \mathrm{n}_{\mathrm{H}}$.

Woods et al. (2017) measured the ionization fraction of the ambient hydrogen ahead of the forward shock to be $\chi_{\mathrm{e}}<0.2$ (the ambient hydrogen is more than $80 \%$ neutral). They obtained the ionization fraction for the atomic gas, which has a higher density; they used $n_{\mathrm{H}}=1 \mathrm{~cm}^{-3}$. Setting $\chi_{\mathrm{e}}=0.2=\frac{n_{\mathrm{e}}}{n_{\mathrm{H}}}$ means that the cavity must be very small, $l_{\text {cav }}<0.02 \mathrm{pc}$.

As mentioned above, a thin length for $l_{\text {cav }}$ is supported by the geometry of the external absorption map, which appears to be limb-brightened. However, this is a very restrictive value, requiring that Tycho be almost, but not quite, interacting with the molecular cloud, and not just in one place but around its entire perimeter. This is very unlikely.

3. Ionized dense molecular environment surrounding Tycho. In this section we consider whether the ionized structure is related to the molecular cloud found by Lee et al. (2004) and discussed in Zhou et al. (2016). The morphological coincidence of the molecular cloud in the northeast with the region of highest absorption is suggestive of such a relation.

Zhou et al. (2016) tabulate the molecular hydrogen column density $N_{\mathrm{H}_{2}}$ for several positions and find values around $7 \times$ $10^{20} \mathrm{~cm}^{-2}$ in the area where we measure $\tau_{40, \mathrm{ISM}}=0.65$, implying $E M=0.30 \mathrm{pc} \mathrm{cm}^{-6}$ (here the conditions $Z=1$, $T=10 \mathrm{~K}$ do apply). Since $E M=n_{\mathrm{e}}^{2} l, N_{\mathrm{H}_{2}}=n_{\mathrm{H}_{2}} l$, and $\chi_{\mathrm{e}}=$ $\frac{n_{\mathrm{e}}}{n_{\mathrm{H}_{2}}}$, the value $\frac{E M}{N_{\mathrm{H}_{2}}}=\chi_{\mathrm{e}} n_{\mathrm{e}}=4.3 \times 10^{-4} \mathrm{~cm}^{-3}$ is independent of the size of the molecular cloud.

If we take the size of the molecular clouds to be of the order of Tycho $\left(l_{\mathrm{MC}} \sim 5 \mathrm{pc}\right.$; see Figure 1, bottom right in Zhou et al. 2016), then $n_{\mathrm{e}}=0.25 \sqrt{\frac{l_{\mathrm{MC}}}{5 \mathrm{pc}}} \mathrm{cm}^{-3}$, which corresponds to $\chi_{\mathrm{e}}=$ $2 \times 10^{-3}\left(\frac{l_{\mathrm{MC}}}{5 \mathrm{pc}}\right)^{-1 / 2}$.

Generally, dense molecular cores have $\chi_{\mathrm{e}} \sim 10^{-8}-10^{-6}$ (Caselli et al. 1998), while translucent and diffuse molecular gas has typical $\chi_{\mathrm{e}} \lesssim 10^{-4}$ (Snow \& McCall 2006; Figure 1). $\chi_{\mathrm{e}} \sim 10^{-3}$ requires an external ionizing source.

It is not possible to tell directly from our observations of free-free absorption whether the ionized absorbing component is in the environs of Tycho or far in the ISM along the line of sight. However, the fact that the absorption occurs where the remnant is brighter and expanding into a higher density region (Reynoso et al. 1999; Williams et al. 2013) is suggestive to us of a local effect, as is the rimmed geometry. If the thin cavity surrounding Tycho and separating the SNR shock from the molecular ring were responsible for the absorption, then the cavity would have to be very thin but at the same time the shock could not have reached the molecular material anywhere along its boundary - a contrived geometry. The high neutral values inferred by Woods et al. (2017), the clear presence of Balmer shocks (Ghavamian et al. 2000), and the morphological coincidence with the molecular cloud in the northeast all point toward the molecular material being associated with the absorption. Finally, the bubble-like distribution of the molecular gas provides a natural explanation for the rimmed absorption morphology. We conclude that the absorption is most likely due to the presence of over-ionized molecular clouds.

\subsection{What Mechanism is Responsible for the Ionization of Tycho's Surroundings?}

A SIMBAD query toward the direction of Tycho gives no OB associations or bright stars that could be responsible for the observed ionization: Tycho itself is the only likely ionizing source toward this line of sight. The sources of ionization could be the X-ray emission from Tycho, the cosmic rays accelerated in the SNR, or perhaps the ionizing radiation emitted by the supernova progenitor or the event itself. A full discussion of the different ionization scenarios requires a detailed treatment of ionization and recombination in the modeling, and is beyond the scope of this paper. 


\subsection{Internal Absorption and Mass in the Unshocked Ejecta}

The amount of mass in ionized material internal to the SNR reverse shock is given by (see Arias et al. 2018)

$$
M=A S l^{1 / 2} m_{\mathrm{p}} \frac{1}{Z} \sqrt{E M},
$$

where $A$ is the mass number of the ions, $S$ is the area of the region for which we measure the absorption, $l$ is the depth of the absorbing material, $m_{\mathrm{p}}$ is the mass of the proton, $Z$ is the number of charges, and $E M$ is the emission measure. Making certain assumptions about these values, one can derive a value for the mass in unshocked material from our measured optical depth.

The easiest parameter to estimate is the mass number of the ions $A$. Tycho is the result of a Type Ia explosion; out of the $\sim 1.4 M_{\odot}$ of ejecta it produced, $0.5-0.8 M_{\odot}$ is expected to be iron (Badenes et al. 2006). In a spectroscopic analysis of the Advanced Satellite for Cosmology and Astrophysics (ASCA) data Hwang et al. (1998) noted that iron is in fact the most recently ionized element, and so it is likely to compose the bulk of the unshocked material. Hayato et al. (2010) also found segregation of $\mathrm{Fe}$ in the inner ejecta from a study of the expansion velocities of the X-ray emitting material. Moreover, the X-ray emission from iron in Tycho is not as prominent as in other type Ia SNRs (e.g., Kepler, Reynolds et al. 2007), suggesting that some of it is not visible in the X-rays yet. For these reasons we take $A=56$, corresponding to Fe. We take $Z=3$, for three times ionized Fe.

$S$ is the surface area of the absorbing region (the area in yellow in Figure 6). We do not know the thickness of the absorbing slab $l$, which is actually critical for the mass determination, because we do not have a way of probing the three-dimensional structure of the absorbing material. For a homogeneous distribution of material within the sphere of the reverse shock, the average depth is $l=\frac{4}{3} R$ (where $R$, the radius of the reverse shock, is $2.25 \mathrm{pc}$ for a distance of $2.5 \mathrm{kpc}$; Tian \& Leahy 2011).

Finally, the value of the $E M$ depends on $Z$ and the temperature $T$. We do not know what the temperature conditions in the unshocked ejecta of Tycho are; an accurate determination would require infrared observations that could measure the ratios between different forbidden lines of the ionized material. To our knowledge, the only time the temperature from the unshocked ejecta of a SNR has been measured is in the case of Cas A, whose unshocked ejecta has a temperature of $100 \mathrm{~K}$ (Raymond et al. 2018). Although it is not clear that the radiation from Tycho's SNR could maintain its internal material heated to $100 \mathrm{~K}$, we will take this to be the value in our mass estimate.

The $E M$ values in Table 2 correspond to the following mass estimates:

$$
\begin{aligned}
M= & 6.5 \pm 2.1 M_{\odot}\left(\frac{A}{56}\right)\left(\frac{l}{3.0 \mathrm{pc}}\right)^{1 / 2}\left(\frac{Z}{3}\right)^{-3 / 2} \\
& \left(\frac{T}{100 \mathrm{~K}}\right)^{3 / 4} \times \sqrt{\frac{g_{\mathrm{ff}}(T=100 \mathrm{~K}, Z=3)}{g_{\mathrm{ff}}(T, Z)}},
\end{aligned}
$$

in the case of the upper limit with $E M=0.33 \mathrm{pc} \mathrm{cm}^{-6}$, and in the case of $E M=179 \mathrm{pc} \mathrm{cm}^{-6}, M=146 \pm 39 M_{\odot}$, with the same parameterization.

\subsection{What are the Conditions and Structure of the Ejecta Internal to Tycho's Reverse Shock?}

Our upper limit above is not useful, and the mass estimate for the $\alpha=0.71$ fit is completely unreasonable, since the total amount of ejecta resulting from the explosion of Tycho's progenitor was $\sim 1.4 M_{\odot}$. As we mention above, a determination of the $E M$ depends very much on the expected flux if no absorption were present, but if there is indeed absorption noticeable at LOFAR HBA frequencies $(\sim 150 \mathrm{MHz})$, then the high-mass estimate value implies that the conditions we assumed in the section above do not describe the actual physical conditions internal to the SNR reverse shock.

Lowering the temperature or invoking a higher ionization state alone are not sufficient to arrive at a meaningful mass estimate. A further way to reduce the mass estimate for a given $E M_{\text {int }}$ is if not all unshocked material is iron, but lighter elements are also present. Decourchelle (2017) notes that the comparison of iron-L complex and $\mathrm{Si}-\mathrm{K}$ line images indicates good mixing of the $\mathrm{Si}$ and $\mathrm{Fe}$ layers synthesized in the supernova. The mass number of $\mathrm{Si}$ is half of that of $\mathrm{Fe}$, so if silicon is present, the mass estimate could be significantly reduced.

The effects of temperature, ionization conditions, and composition can be important if combined, but the single effect that can have the largest contribution to the high absorption value is the degree of clumping in the unshocked material. The estimate in Equation (9) assumes that the ejecta are distributed homogeneously within the sphere of the reverse shock. This is what one expects for an ejecta density profile with a flat core and an exponential outer region (Chevalier 1982), if the reverse shock has already reached the core.

Sato et al. (2019) analyzed Chandra observations of Tycho and found from its genus statistic that Tycho's X-ray ejecta structure strongly indicates a skewed non-Gaussian distribution of the ejecta clumps, possibly from initially clumped ejecta. The radioactive decay of elements synthesized in the explosion could also cause the ejecta to have a foamy distribution, as is the case for Cas A (Milisavljevic \& Fesen 2015). If the unshocked ejecta in Tycho are heavily clumped it can be possible to see absorption in the LOFAR HBA even for modest amounts of unshocked mass.

\section{Conclusions}

In this work we have mapped Tycho's SNR with the LOFAR LBA and HBA, centered at $58 \mathrm{MHz}$ and $143 \mathrm{MHz}$, respectively. These are the lowest-frequency resolved observations of this source to date, even though the angular resolution of our LBA maps is modest $\left(41^{\prime \prime}\right)$. We compared these maps to higher frequency VLA observations at 330 and $1400 \mathrm{MHz}$ (Katz-Stone et al. 2000; Williams et al. 2016) and found that in some regions the LOFAR flux is lower than expected for an unabsorbed synchrotron source. We identify this effect as lowfrequency free-free absorption due to foreground free electrons absorbing the background synchrotron radiation from Tycho.

It is unlikely, from the observed geometry, that the lowfrequency absorption is due to line-of-sight material far away from Tycho, but rather it must be in the environment of the SNR. There are two regions that could be responsible for the ionization: the diffuse, infrared-emitting region immediately surrounding Tycho, or its neighboring molecular clouds. If the former is true, and the absorption is due to an ionized 
cavity surrounding Tycho, then this cavity must be very thin $(<0.02 \mathrm{pc})$, so as to not contradict earlier results on the neutral fraction ahead of the shock. Alternatively, if the molecular clouds are responsible for the absorption, then the implied ionization fraction requires an external ionizing source. Tycho itself is the only candidate, through its X-ray emission, its cosmic rays, or possibly from the ionizing flux of its progenitor white dwarf or the supernova explosion.

Finally, we tried to measure the free-free absorption in the region internal to the SNR reverse shock from its unshocked ejecta. However, we are limited by our knowledge of the unabsorbed spectral behavior of the source at these frequencies: the amount of absorption we measure depends on what is the spectral index in the region, which is poorly constrained due to systematic error and an incomplete knowledge of the spectral behavior at high frequencies. According to our best-fit scenario, the spectral index in the region internal to the reverse shock is relatively high and a copious amount of free-free absorption is required to explain the LOFAR flux densities. If real, we attribute the absorption to cold, ionized, unshocked stellar ejecta inside the SNR reverse shock free-free absorbing the synchrotron emission from the back side of the shell. In order to account for the high value of internal absorption we measure, we expect the ejecta to be colder than $100 \mathrm{~K}$, be somewhat highly ionized, and be heavily clumped.

Radio observations in the few gigahertz range could determine the unabsorbed, resolved spectral index of the source, and observations in the $200-1000 \mathrm{MHz}$ range would allow us to better model the parameters responsible for the absorption, which result in a characteristic spectrum with curvature at these frequencies. Finally, hyperfine, structured, infrared line observations of these clumps would be necessary to better understand their temperature and composition, both critical in determining the mass in unshocked ejecta.

We thank N. Kassim for the $330 \mathrm{MHz}$ VLA image and B. Williams for the $1.4 \mathrm{MHz}$ VLA image.

This paper is based (in part) on data obtained with the International LOFAR Telescope (ILT) under project code LC10_011. LOFAR (van Haarlem et al. 2013) is the low frequency array designed and constructed by ASTRON. It has observing, data processing, and data storage facilities in several countries, that are owned by various parties (each with their own funding sources), and that are collectively operated by the ILT foundation under a joint scientific policy. The ILT resources have benefited from the following recent major funding sources: CNRS-INSU, Observatoire de Paris and Université d'Orléans, France; BMBF, MIWF-NRW, MPG, Germany; Science Foundation Ireland (SFI), Department of Business, Enterprise and Innovation (DBEI), Ireland; NWO, The Netherlands; The Science and Technology Facilities Council, UK.

We acknowledge the use of archival data from the National Radio Astronomy Observatory's Karl G. Jansky Very Large Array (VLA). The National Radio Astronomy Observatory is a facility of the National Science Foundation operated under cooperative agreement by Associated Universities, Inc.

Software: LOFAR Low-Frequency Pipeline (de Gasperin et al. 2019), wsclean (Offringa et al. 2014), Pre-Facet Calibration Pipeline (van Weeren et al. 2016), ddf-pipeline (v2.2; Shimwell et al. 2019), LMFIT: Non-Linear Least-Square Minimization and Curve-Fitting for Python (Newville et al. 2014), APLpy:
Astronomical Plotting Library in Python (Robitaille \& Bressert 2012).

Facilities: The LOw Frequency ARray (LOFAR), the Karl G. Jansky Very Large Array (VLA).

\section{ORCID iDs}

Maria Arias (ㄴ) https://orcid.org/0000-0002-7918-904X

Jacco Vink ํㅜ https://orcid.org/0000-0002-4708-4219

\section{References}

Arias, M., Vink, J., de Gasperin, F., et al. 2018, A\&A, 612, A110

Artyukh, V. S., Dagkesamanskii, V. V., Vitkevich, R. D., \& Kozhukhov, V. N. 1969, SvA, 12, 567

Baade, W. 1943, ApJ, 97, 119

Badenes, C., Borkowski, K. J., Hughes, J. P., Hwang, U., \& Bravo, E. 2006, ApJ, 645, 1373

Bennett, A. S. 1963, MNRAS, 127, 3

Briggs, D. S. 1995, AAS Meeting, 187, 112.02

Caselli, P., Walmsley, C. M., Terzieva, R., \& Herbst, E. 1998, ApJ, 499, 234

Chevalier, R. A. 1982, ApJ, 258, 790

Conway, R. G., Daintree, E. J., \& Long, R. J. 1965, MNRAS, 131, 159

de Gasperin, F., Dijkema, T. J., Drabent, A., et al. 2019, A\&A, 622, A5

Decourchelle, A. 2017, in Handbook of Supernovae, ed. A. Alsabti \& P. Murdin (Cham: Springer), 117

Draine, B. T. 2011, Physics of the Interstellar and Intergalactic Medium (Princeton, NJ: Princeton Univ. Press)

Duin, R. M., \& Strom, R. G. 1975, A\&A, 39, 33

Fanti, C., Felli, M., Ficarra, A., et al. 1974, A\&AS, 16, 43

Gao, X. Y., Han, J. L., Reich, W., et al. 2011, A\&A, 529, A159

Ghavamian, P., Raymond, J., Hartigan, P., \& Blair, W. P. 2000, ApJ, 535, 266

Gomez, H. L., Clark, C. J. R., Nozawa, T., et al. 2012, MNRAS, 420, 3557

Green, A. J., Baker, J. R., \& Landecker, T. L. 1975, A\&A, 44, 187

Green, D. A. 2017, yCat, VII/278, 0

Hayato, A., Yamaguchi, H., Tamagawa, T., et al. 2010, ApJ, 725, 894

Hillas, A. M. 2005, JPhG, 31, R95

Horton, P. W., Conway, R. G., \& Daintree, E. J. 1969, MNRAS, 143, 245

Hurley-Walker, N., Scaife, A. M. M., Green, D. A., et al. 2009, MNRAS, 396,365

Hwang, U., Decourchelle, A., Holt, S. S., \& Petre, R. 2002, ApJ, 581, 1101

Hwang, U., Hughes, J. P., \& Petre, R. 1998, ApJ, 497, 833

Kassim, N. E. 1989, ApJ, 347, 915

Katz-Stone, D. M., Kassim, N. E., Lazio, T. J. W., \& O’Donnell, R. 2000, ApJ, 529,453

Kellermann, K. I., Pauliny-Toth, I. I. K., \& Williams, P. J. S. 1969, ApJ, 157, 1

Klein, U., Emerson, D. T., Haslam, C. G. T., \& Salter, C. J. 1979, A\&A, 76,120

Kothes, R., Fedotov, K., Foster, T. J., \& Uyanıker, B. 2006, A\&A, 457, 1081

Krause, O., Tanaka, M., Usuda, T., et al. 2008, Natur, 456, 617

Langston, G., Minter, A., D’Addario, L., et al. 2000, AJ, 119, 2801

Lee, R. E., Chapman, S. C., \& Dendy, R. O. 2004, ApJ, 604, 187

Lopez, L. A., Grefenstette, B. W., Reynolds, S. P., et al. 2015, ApJ, 814, 132

McKee, C. F. 1974, ApJ, 188, 335

Milisavljevic, D., \& Fesen, R. A. 2015, Sci, 347, 526

Newville, M., Stensitzki, T., Allen, D. B., \& Ingargiola, A. 2014, LMFIT: NonLinear Least-Square Minimization and Curve-Fitting for Python, 0.8.0, Zenodo, doi:10.5281/zenodo.11813

Offringa, A. R., McKinley, B., Hurley-Walker, N., et al. 2014, MNRAS, 444, 606

Planck Collaboration, Arnaud, M., Ashdown, M., et al. 2016, A\&A, 586, A134 Raymond, J. C., Koo, B. C., Lee, Y. H., et al. 2018, ApJ, 866, 128

Rest, A., Welch, D. L., Suntzeff, N. B., et al. 2008, ApJL, 681, L81

Reynolds, S. P., Borkowski, K. J., Hwang, U., et al. 2007, ApJL, 668, L135

Reynolds, S. P., \& Ellison, D. C. 1992, ApJL, 399, L75

Reynoso, E. M., Moffett, D. A., Goss, W. M., et al. 1997, ApJ, 491, 816

Reynoso, E. M., Velázquez, P. F., Dubner, G. M., \& Goss, W. M. 1999, AJ, 117,1827

Robitaille, T., \& Bressert, E. 2012, APLpy: Astronomical Plotting Library in Python, Astrophysics Source Code Library, ascl:1208.017

Roger, R. S., Costain, C. H., \& Bridle, A. H. 1973, AJ, 78, 1030

Rybicki, G. B., \& Lightman, A. P. 1979, Radiative Processes in Astrophysics (New Yor: Wiley)

Sato, T., Hughes, J. P., Williams, B. J., \& Morii, M. 2019, ApJ, 879, 64

Scott, P. F., \& Shakeshaft, J. R. 1971, MNRAS, 154, 19P 
Shimwell, T. W., Röttgering, H. J. A., Best, P. N., et al. 2017, A\&A, 598, A104

Shimwell, T. W., Tasse, C., Hardcastle, M. J., et al. 2019, A\&A, 622, A1

Smirnov, O. M., \& Tasse, C. 2015, MNRAS, 449, 2668

Snow, T. P., \& McCall, B. J. 2006, ARA\&A, 44, 367

Tasse, C. 2014a, arXiv: 1410.8706

Tasse, C. 2014b, A\&A, 566, A127

Tasse, C., Hugo, B., Mirmont, M., et al. 2018, A\&A, 611, A87

Tian, W. W., \& Leahy, D. A. 2011, ApJL, 729, L15

van Haarlem, M. P., Wise, M. W., Gunst, A. W., et al. 2013, A\&A, 556, A2

van Weeren, R. J., Williams, W. L., Hardcastle, M. J., et al. 2016, ApJS, 223, 2
Vinyaikin, E. N., Volodin, Y. V., Dagkesamanskii, R. D., \& Sokolov, K. P. 1987, SvA, 31, 141

Warren, J. S., et al. 2005, ApJ, 634, 376

Williams, B. J., Borkowski, K. J., Ghavamian, P., et al. 2013, ApJ, 770, 129

Williams, P. J. S., Kenderdine, S., \& Baldwin, J. E. 1966, MmRAS, 70, 53

Williams, W. L., van Weeren, R. J., Röttgering, H. J. A., et al. 2016, MNRAS, 460, 2385

Woods, T. E., Ghavamian, P., Badenes, C., \& Gilfanov, M. 2017, NatAs, 1,800

Zhou, P., Chen, Y., Zhang, Z.-Y., et al. 2016, ApJ, 826, 34 